\title{
Natural frequencies of a cracked beam coupled with a compressible sloshing fluid
}

\author{
Michele Di Sciuva ${ }^{\mathrm{a}}$ and Cecilia Surace ${ }^{\mathrm{b}, *}$ \\ ${ }^{a}$ Department of Aeronautical and Space Engineering, Politecnico di Torino, Corso Duca degli Abruzzi 24, 10129, \\ Torino, Italy \\ ${ }^{\mathrm{b}}$ Department of Structural and Geotechnical Engineering, Politecnico di Torino, Corso Duca degli Abruzzi 24, \\ 10129, Torino, Italy
}

Caro Prof. Piombo,

Ti voglio ringraziare in modo speciale:

Se non mi avessi mandato a studiare all' Università di Manchester non avrei conosciuto David adesso non ci sarebbero MariaChiara, Julia ed Elizabeth, le mie bellissime bambine! Cecilia

\begin{abstract}
This article describes studies into the flexural vibration of a cracked cantilevered beam in contact with a non-viscous fluid. The crack has been represented by a mass-less rotational spring, the flexibility of which has been calculated using linear fracture mechanics. The coupled system is subject to undisturbed boundary condition at infinity in the fluid domain. A range of different boundary conditions have been analysed such as both incompressible and compressible fluid, with and without sloshing. Various crack sizes and positions have been considered in order to assess the effect of damage in the fluid-structure interaction problem.
\end{abstract}

\section{Introduction}

This article presents the results of research being conducted regarding the interaction of a cracked beam with a surrounding non-viscous, irrotational fluid medium, a topic of direct significance and potential importance for a wide range of practical applications, for example in offshore and irrigation engineering fields. Specifically, in the analysis of offshore platforms and water dams, mathematical models are often simplified by assuming that certain parts of the structure can be considered to be represented by beams in order to address issues related to the interaction with the water.

The research presented in this article essentially incorporates recent developments from diverse areas of research, specifically as concerning fluid-structure interaction and the dynamics of damaged structural components; correspondingly the innovation relating principally to the introduction of a cracked beam into the analysis in order to highlight the potential effect on the coupled frequencies of the structural system.

Firstly, as concerns the issues related to damage in structural systems, during the last decade a significant amount of progress has been made, particularly the use of vibration based inspection has been a topic of active research. An extended literature review of damage identification methods can be found in [13], while in [12] a state of the art review of methods developed to deal with cracked structures is presented.

Furthermore, in order to investigate the prevailing effects of damage present in the structure under examination, several studies introduced damage into the mathematical model through a simple reduction of the stiffness on a given zone of the structure $[22,31,42]$.

\footnotetext{
*Corresponding author. E-mail: cecilia.surace@polito.it.
} 
With the scope of investigating the variation in dynamic properties due to the presence of real damage, several papers have evaluated the changes in natural frequencies of a simple cantilever beam due to the presence of cracks [10, $11,15,16,18,27-29,32,33,35-38]$. Moreover, in the same papers, the method for calculating the shift in natural frequencies is also often proposed in order to address the inverse problem.

A second area of fundamental importance for the research presented concerns the coupling between the dynamic behaviour of elastic structures and irrotational non-viscous fluids, an area which has been widely studied in the past with a relatively large body of published literature available which refers to a range of different geometries and structural elements. In particular, the resolution of the fluid-structure interaction problem has been conducted using analytical methods [7,40], semi-analytical methods [1-6,8] and numerical methods [17].

Furthermore, in the past, the influence of the presence of a compressible fluid on the natural frequency of a plate has been subject of numerous studies [26], the results demonstrating quite clearly that the natural frequencies of a plate in contact with the fluid are lower with respect to the same plate in a vacuum.

The review of existing literature reveals that the main part of articles considers the behaviour of circular plates. Among these [4] used the Hankel trasform to obtain the so-called NAMVI factors for a circular plate on the free surface of a liquid of infinite domain; studied [5,9] the influence of the Poisson ratio on NAMVI for the same geometry, examining the asymmetric vibrations of the elastic bottom of a cylindrical container and considering also the effects of an elastic foundation.

In comparison, the number of works related to rectangular plates is very limited Bauer [7] studied the vibrations of a simply supported elastic bottom of a prismatic container, while performed research regarding the vibrations of rectangular clamped plates immersed in a fluid Kwak [24] determined the NAMVI factors for rectangular plates in contact with a fluid of infinite domain, analysed the behaviour of an isotropic rectangular plate in contact with a liquid domain, and in $[14,30]$ the case of a rectangular plate clamped in the rigid bottom of a prismatic container has been considered.

Nevertheless these works have not taken into account the presence of a fluid with free-surface overhanging an elastic plate and, concerning this issue, the number of articles published is limited [25] described the behaviour of liquid films on a stationary structure rapoport [34] formulated the equations for elastic bodies containing fluids, but did not provide detailed solutions for specific cases. The work described in [23] considered the case of simply supported rectangular plates that constituted a portion of the container wall Soedel [39] studied the free oscillations of a beam in contact with a liquid with a free-surface.

Research related to elastic structures coupled to compressible fluids is the subject of even smaller numbers of published articles. In [19-21] the exact solution for the dynamics of couples of concentric and eccentric cylinders immersed in a compressible fluid was determined, while taking into account a boundary condition of free surface of the fluid. In [41] the coupled dynamics of a basin of infinite dimensions with an elastic beam was analysed while considering the compressibility of the fluid.

The present work analyses the flexural behaviour of a cracked dam modelled with a prismatic beam in contact with a compressible fluid with free surface, the crack being represented by a massless rotational spring. Following the approach of [41] the coupled beam-water eigen-problem has been solved using the method of variable-separation. An exact solution has been obtained for several boundary conditions: an incompressible fluid (Laplace problem) without sloshing, a compressible fluid without sloshing, and a compressible fluid (Helmholtz problem) with sloshing. Different crack sizes and positions have been considered in order to assess the effect of damage in the fluid-structure interaction problem.

\section{Formulation of the problem}

\subsection{Generalities}

An elastic beam (Fig. 1) with transversal section $A$, length $L$, moment of inertia $J$, Young's modulus $E$ and density $\rho_{S}$ has been considered. The beam is characterised by a one-edge non-propagating transversal crack of length $a$ at distance $L_{1}$ from the clamped end. The beam is in contact with an irrotational, non viscous fluid of $\rho_{F}$ density inside of which the speed of sound is $c$. The height of the free surface is $L_{2}$ and the fluid occupies a spatially unlimited region in the opposite direction of the beam. The equations governing the phenomenon under the hypothesis of small displacements of the beam and small variations of pressure are determined as described in the following sections. 


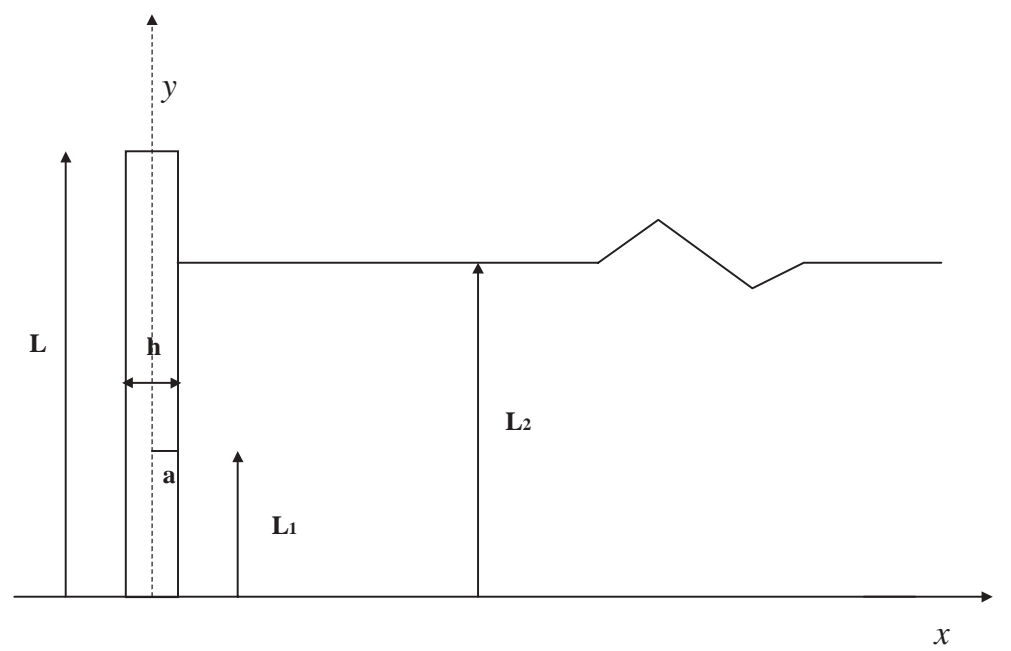

Fig. 1. The coupled system: a cracked beam interacting with a free surface fluid.

\subsection{Equations of the fluid}

The pressure of the fluid can be expressed by the following equation:

$$
\frac{\partial^{2} p}{\partial x^{2}}+\frac{\partial^{2} p}{\partial y^{2}}=\frac{1}{c^{2}} \frac{\partial^{2} p}{\partial t^{2}}
$$

valid for $0<x<\infty, 0<y<L_{2}$

The boundary conditions of the problem are:

$$
P=\varnothing, y=\varnothing \quad(\text { Free Surface Wave Not Included })
$$

$$
\frac{\partial p}{\partial y}=-\frac{1}{g} \frac{\partial^{2} p}{\partial t^{2}} \text { (Sloshing) }
$$

for $y=L_{2}$, being $g$ the acceleration of gravity.

On the bottom of the reservoir the following condition is valid:

$$
\frac{\partial p}{\partial y}=0
$$

for $y=0$.

As the fluid domain is not limited, it is assumed that the pressure disturbance does not transmit to infinity in the water; namely there is an undisturbed condition expressed as follows:

$$
\begin{aligned}
& p=0 \\
& x \rightarrow \infty
\end{aligned}
$$

\subsection{Equations of the beam}

The equation of motion for the cracked beam, according to Euler-Bernoulli theory is:

$$
E J\left(\frac{\partial^{4} u_{1}}{\partial y^{4}}\right)+\rho_{s} A\left(\frac{\partial^{2} u_{1}}{\partial t^{2}}\right)=-p(0, y, t) \text { for } 0<y<L_{1}
$$




$$
\begin{aligned}
& E J\left(\frac{\partial^{4} u_{2}}{\partial y^{4}}\right)+\rho_{s} A\left(\frac{\partial^{2} u_{2}}{\partial t^{2}}\right)=-p(0, y, t) \text { for } L_{1}<y<L_{2} . \\
& E J\left(\frac{\partial^{4} u_{3}}{\partial y^{4}}\right)+\rho_{s} A\left(\frac{\partial^{2} u_{3}}{\partial t^{2}}\right)=0 \text { for } L_{2}<y<L .
\end{aligned}
$$

where $L_{1}$ and $L_{2}$ are the positions of the crack and of the liquid free surface, respectively, and $u_{1}$ is the transversal displacement.

For this problem the following boundary conditions must be respected:

$$
\begin{aligned}
u_{1}(0, t) & =0 \\
\frac{\partial u_{1}(0, t)}{\partial y} & =0
\end{aligned}
$$

at the clamped end and

$$
\begin{aligned}
& \frac{\partial^{3} u_{3}(L, t)}{\partial y^{3}}=0 \\
& \frac{\partial^{2} u_{3}(L, t)}{\partial y^{2}}=0
\end{aligned}
$$

at the free end.

Furthermore the following conditions of continuity on displacement, rotation, shear and bending moment must be guaranteed in correspondence of the free surface:

$$
\begin{gathered}
u_{2}\left(L_{2}, t\right)=u_{3}\left(L_{2}, t\right) \\
\frac{\partial u_{2}\left(L_{2}, t\right)}{\partial y}=\frac{\partial u_{3}\left(L_{2}, t\right)}{\partial y} \\
\frac{\partial^{2} u_{2}\left(L_{2}, t\right)}{\partial y^{2}}=\frac{\partial^{2} u_{3}\left(L_{2}, t\right)}{\partial y^{2}} \\
\frac{\partial^{3} u_{2}\left(L_{2}, t\right)}{\partial y^{3}}=\frac{\partial^{3} u_{3}\left(L_{2}, t\right)}{\partial y^{3}}
\end{gathered}
$$

and the following conditions in correspondence with the cracked section:

$$
\begin{aligned}
u_{1}\left(L_{1}, t\right) & =u_{2}\left(L_{1}, t\right) \\
\frac{\partial^{2} u_{1}\left(L_{1}, t\right)}{\partial y^{2}} & =\frac{\partial^{2} u_{2}\left(L_{1}, t\right)}{\partial y^{2}} \\
\frac{\partial^{3} u_{1}\left(L_{1}, t\right)}{\partial y^{2}} & =\frac{\partial^{3} u_{2}\left(L_{1}, t\right)}{\partial y^{2}} \\
\frac{\partial u_{1}\left(L_{1}, t\right)}{\partial y}-\frac{\partial u_{2}\left(L_{1}, t\right)}{\partial y} & =\frac{E J}{k_{T}} \frac{\partial^{2} u_{1}\left(L_{1}, t\right)}{\partial y^{2}}
\end{aligned}
$$

As only the transversal oscillations of the beam are considered, it is possible to model the crack according to [35] where the stiffness of the spring representative of the crack has been evaluated in the following way:

$$
\begin{aligned}
& k_{T}=\frac{1}{c_{T}} \\
& c_{T}=\frac{6\left(1-\nu^{2}\right) h}{E J} f\left(\frac{a}{h}\right)
\end{aligned}
$$

where $h$ is the height of the transversal section of the beam, $\nu$ is the Poisson's ratio, and $f\left(\frac{a}{h}\right)$ has the following expression: 


$$
\begin{aligned}
f\left(\frac{a}{h}\right)= & 1.8264\left(\frac{a}{h}\right)^{2}-3.95\left(\frac{a}{h}\right)^{3}+16.375\left(\frac{a}{h}\right)^{4}-37.266\left(\frac{a}{h}\right)^{5}+76.81\left(\frac{a}{h}\right)^{6}-126.9\left(\frac{a}{h}\right)^{7} \\
& +172\left(\frac{a}{h}\right)^{8}-143.97\left(\frac{a}{h}\right)^{9}+66.56\left(\frac{a}{h}\right)^{10}
\end{aligned}
$$

\subsection{Fluid-structure interaction}

At the fluid-structure interface, the pressure of the water and the displacement field are related as follows:

$$
\frac{\partial p}{\partial x}=-\rho_{F} \frac{\partial^{2} u_{1}}{\partial t^{2}}
$$

for

$$
x=0 ; \quad 0<y<L_{1}
$$

and:

$$
\begin{aligned}
& \frac{\partial p}{\partial x}=-\rho_{F} \frac{\partial^{2} u_{2}}{\partial t^{2}} \\
& x=0 ; \quad L_{1}<y<L_{2}
\end{aligned}
$$

\subsection{The proposed solution}

Using the variable separation it is possible to write:

$$
\begin{aligned}
p(x, y, t) & =f_{x}(x) f_{y}(y) f_{t}(t) \\
u_{1}(y, t) & =u_{1}(y) f_{t}(t) \\
u_{2}(y, t) & =u_{2}(y) f_{t}(t) \\
u_{3}(y, t) & =u_{3}(y) f_{t}(t)
\end{aligned}
$$

Substituting Eqs (7) in Eq. (1) and in the boundary conditions the three functions $f_{x}(x) f_{y}(y) f_{t}(t)$ are obtained as follows:

$$
f_{t}^{\prime \prime}+\Omega^{2} f_{t}=0
$$

with solution:

$$
f_{t}(t)=a_{1} \cos \Omega t+a_{2} \sin \Omega t
$$

Then, for the variable that expresses the dependence of the pressure on $y$ :

$$
f_{y}^{\prime \prime}+k^{2} f_{y}=0
$$

with the following conditions derived by Eqs (2a,b,c):

$$
f_{y}^{\prime}(0)=0
$$

$$
f_{y}\left(L_{2}\right)=0 \quad \text { (free surface waves not included) }
$$

$$
f_{y}^{\prime}\left(L_{2}\right)-\left(\Omega^{2} / g\right) f_{y}\left(L_{2}\right)=0 \text { (free surface waves included) }
$$


The solution is:

$$
f_{y}(y)=a_{3} \cos k y
$$

Finally, for the spatial variable that expresses the dependence of the pressure on $x$ :

$$
f_{x}^{\prime \prime}-\lambda^{2} f_{x}=0
$$

with:

$$
\begin{aligned}
& f_{x}(x)=0 \\
& x \rightarrow \infty
\end{aligned}
$$

and solution derived from Eq. (2d):

$$
f_{x}(x)=a_{4} e^{-\lambda x}
$$

In the Eqs $(8,9,10)$ the unknown parameters $\lambda, k, \Omega$ are real and are linked by the following relationship:

$$
\lambda^{2}=\left(k^{2}-\frac{\Omega^{2}}{c^{2}}\right) \text {. }
$$

\section{The eigenvalue problem}

\subsection{Free surface wave neglected}

In the case in which the surface wave is not considered, the boundary conditions Eq. (9c) are satisfied when

$$
k_{n}=\frac{(2 n-1) \pi}{2 L_{2}}, n=1,2 \ldots
$$

At the same time, as $\lambda$ in Eq. (10c) is real, the following inequality must be respected:

$$
\lambda_{n}^{2}=\left(k n^{2}-\frac{\Omega^{2}}{c^{2}}\right)>0
$$

\subsection{Free surface wave considered}

When the surface wave is included, the boundary conditions Eq. (9d) are satisfied when

$$
\tan \left(k_{n} L_{2}\right)=-\frac{\Omega^{2}}{g k_{n}}, \quad n=1,2, \ldots
$$

and contemporarily the inequality Eq. (13) has to be respected.

\subsection{Determinantal equation}

In this paragraph it is shown how the frequencies of the coupled system are obtained through the resolution of a classical eigenvalue problem associated at the annulment of the determinant.

If $n_{1}$ is the minimum positive integer so that inequality Eq. (13) is satisfied, using the superposition principle it is possible to write:

$$
f_{x}(x) f_{y}(y)=\sum_{n=n_{1}}^{\infty} G_{n} e^{-\lambda_{n} x} \cos \left(k_{n} y\right)
$$

with $G_{n}$ real unknown.

Considering the following adimensional variables: 


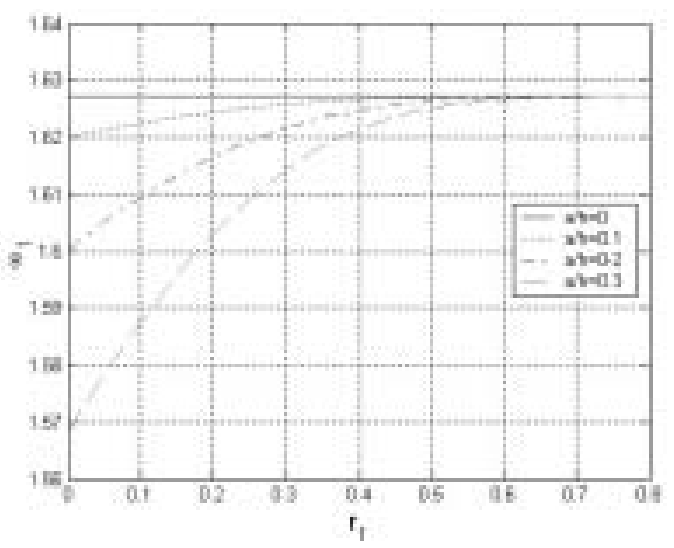

Fig. 2. The first structural frequency parameter of the coupled system as a function of the position of the crack. The fluid is considered to be incompressible without sloshing.

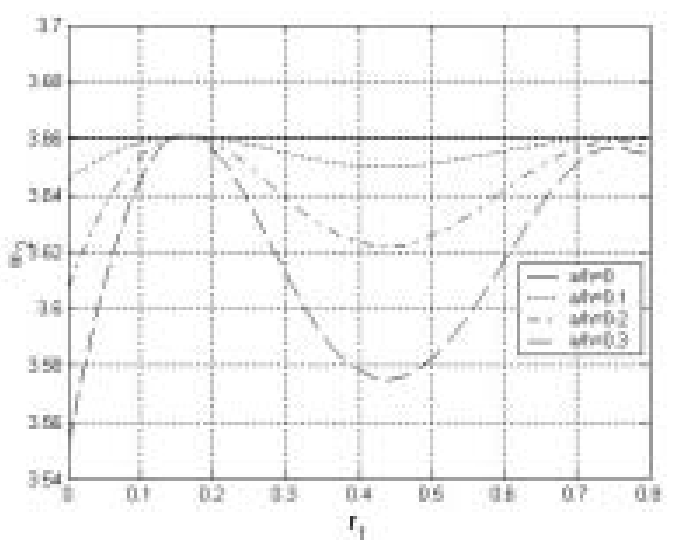

Fig. 3. The second structural frequency parameter of the coupled system as a function of the position of the crack. The fluid is considered to be incompressible without sloshing.

$$
\xi=\frac{y}{L} ; \quad r_{1}=\frac{L_{1}}{L} ; \quad r_{2}=\frac{L_{2}}{L} ; \quad \gamma=\frac{\rho_{F} L}{\rho_{s} A} ; \omega^{2}=\frac{\Omega}{\Omega_{b}} ; \bar{c}=\frac{c}{\Omega_{b} L} ; \quad \bar{k}_{n}=k_{n} L ; \quad \bar{\lambda}_{n}^{2}=\bar{k}_{n}^{2}-\frac{\omega^{4}}{\bar{c}^{2}}
$$

where:

$$
\Omega_{b}=\sqrt{\frac{E J}{L^{4} A \rho_{S}}}
$$

and substituting formulas Eqs (15) and (16) into Eqs $(3,4,6)$, it possible to rewrite the governing equation of the problem in the following way:

$$
\begin{aligned}
& u_{1}^{(I V)}(\xi)-\omega^{4} u_{1}(\xi)=-\sum_{n_{1}}^{\infty} A_{n}^{(1)} \cos \left(\bar{k}_{n} \xi\right) \\
& 0<\xi<r_{1} \\
& u_{2}^{(I V)}(\xi)-\omega^{4} u_{2}(\xi)=-\sum_{n_{1}}^{\infty} A_{n}^{(2)} \cos \left(\bar{k}_{n} \xi\right) \\
& r_{1}<\xi<r_{2}
\end{aligned}
$$




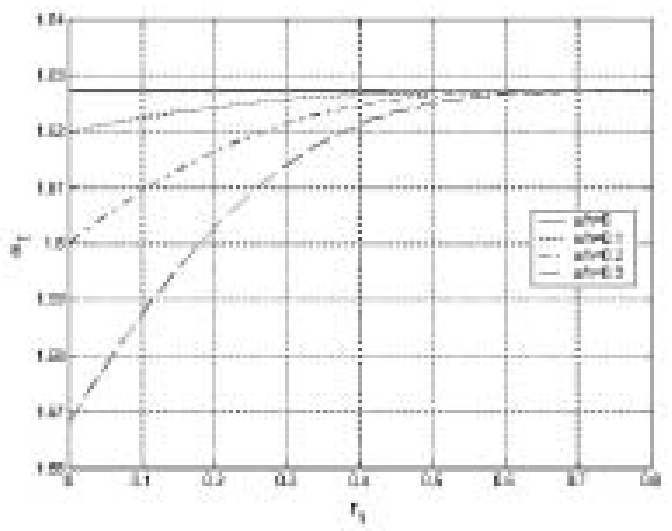

Fig. 4. The first structural frequency parameter of the coupled system as a function of the position of the crack. The fluid is considered compressible without sloshing.

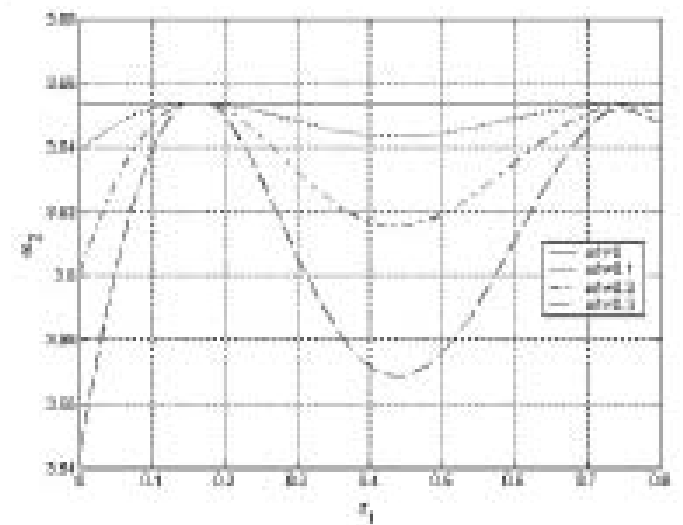

Fig. 5. The second structural frequency parameter of the coupled system as a function of the position of the crack. The fluid is considered to be compressible without sloshing.

$$
\begin{aligned}
& u_{3}^{(I V)}(\xi)-\omega^{4} u_{3}(\xi)=0 \\
& r_{2}<\xi<1
\end{aligned}
$$

with the following boundary conditions:

$$
\begin{aligned}
& u_{1}(0)=0 \\
& u_{1}^{\prime}(0)=0
\end{aligned}
$$

relative to the clamped end and:

$$
\begin{aligned}
u_{1}\left(r_{1}\right) & =u_{2}\left(r_{1}\right) \\
u_{1}^{\prime \prime}\left(r_{1}\right) & =u_{2}^{\prime \prime}\left(r_{1}\right) \\
u_{1}^{\prime \prime \prime}\left(r_{1}\right) & =u_{2}^{\prime \prime \prime}\left(r_{1}\right) \\
u_{1}^{\prime}\left(r_{1}\right)-u_{2}^{\prime}\left(r_{1}\right) & =\frac{E J}{L k_{T}} u_{1}^{\prime \prime}\left(r_{1}\right)
\end{aligned}
$$

relative to the damage section, imposing the continuity of displacement bending moment and shear and the change of the rotation angle due to the presence of the crack. 


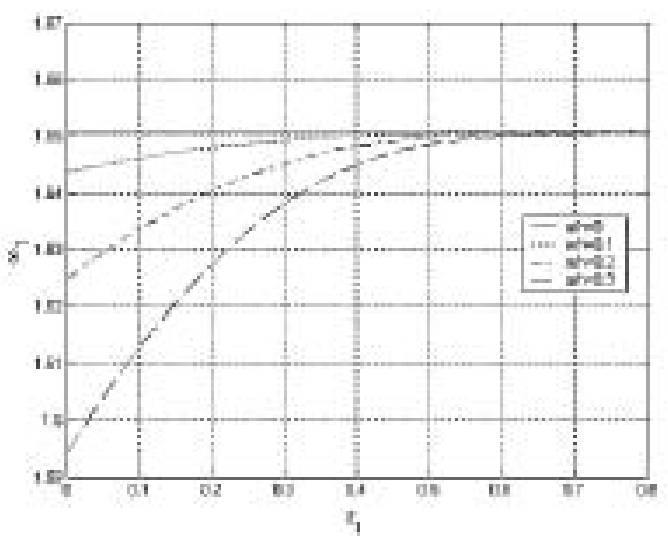

Fig. 6. The first structural frequency parameter of the coupled system as a function of the position of the crack. The fluid is considered to be compressible with sloshing.

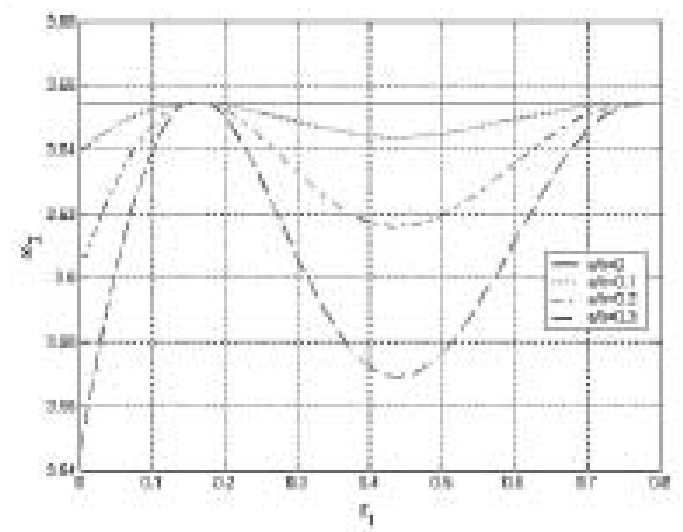

Fig. 7. The second structural frequency parameter of the coupled system as a function of the position of the crack. The fluid is considered to be compressible with sloshing.

Instead the following conditions:

$$
\begin{aligned}
u_{2}\left(r_{2}\right) & =u_{3}\left(r_{2}\right) \\
u_{2}^{\prime}\left(r_{2}\right) & =u_{3}^{\prime}\left(r_{2}\right) \\
u_{2}^{\prime \prime}\left(r_{2}\right) & =u_{3}^{\prime \prime}\left(r_{2}\right) \\
u_{2}^{\prime \prime \prime}\left(r_{2}\right) & =u_{3}^{\prime \prime \prime}\left(r_{2}\right)
\end{aligned}
$$

represent the continuity in the interface section, in correspondence of the free surface of the fluid.

Furthermore the free end conditions must be respected:

$$
\begin{array}{r}
u_{3}^{\prime \prime}(1)=0 \\
u_{3}^{\prime \prime \prime}(1)=0
\end{array}
$$

The equations governing the fluid-structure interaction Eqs $(5,6)$ become:

$$
u_{1}(\xi)=-\sum_{n_{1}}^{\infty} \frac{\bar{\lambda}_{n} A_{n}^{(1)}}{\gamma \omega^{4}} \cos \left(\bar{k}_{n} \xi\right)
$$




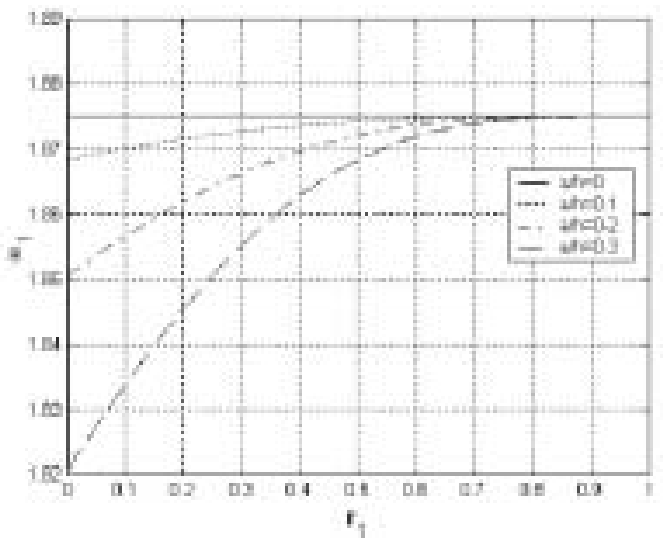

Fig. 8. The first natural frequency parameter of the beam in vacuum as a function of the position of the crack.

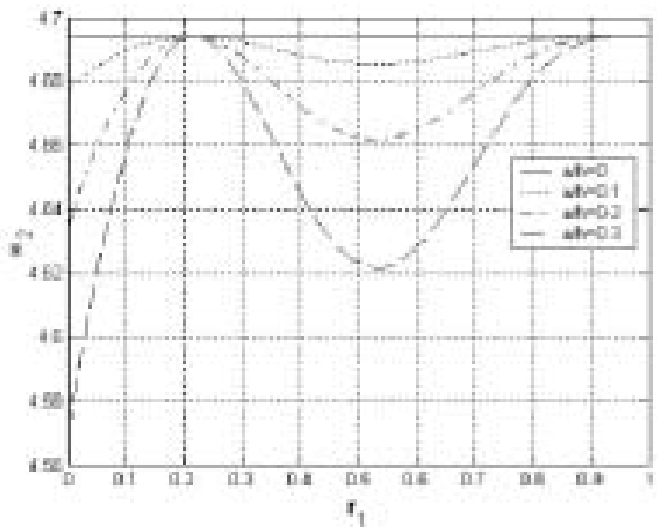

Fig. 9. The second natural frequency parameter of the beam in vacuum as a function of the position of the crack.

$$
\begin{aligned}
& \begin{aligned}
0<\xi & <r_{1} \\
u_{2}(\xi) & =-\sum_{n_{1}}^{\infty} \frac{\bar{\lambda}_{n} A_{n}^{(2)}}{\gamma \omega^{4}} \cos \left(\bar{k}_{n} \xi\right) \\
r_{1}<\xi & <r_{2}
\end{aligned} \\
& \text { here } A_{n}=\frac{G_{n} L^{3}}{E J} \text { is non-dimensional parameter defined for } \\
& \text { The solution of these equations can be obtained as follows: } \\
& u_{1}(\xi)=\sum_{j=1}^{4} D_{j} \phi_{j}(\xi)+\sum_{n=n_{1}}^{\infty} B_{n}^{(1)} \cos \left(\bar{k}_{n} \xi\right) \\
& u_{2}(\xi)=\sum_{j=5}^{8} D_{j} \phi_{j}(\xi)+\sum_{n=n_{1}}^{\infty} B_{n}^{(2)} \cos \left(\bar{k}_{n} \xi\right) \\
& u_{3}(\xi)=\sum_{j=9}^{12} D_{j} \phi_{j}(\xi)
\end{aligned}
$$

where $A_{n}=\frac{G_{n} L^{3}}{E J}$ is non-dimensional parameter defined for every $n_{1} \leqslant n \leqslant \infty$. 
with $B_{n}^{(1),(2)}=\frac{-A_{n}^{(1),(2)}}{\bar{k}_{m}^{4}-\omega^{4}}$

The functions $\phi_{j}$ are defined as:

$$
\begin{aligned}
& \phi_{1}=\cos (\omega \xi) ; \phi_{2}=\sin (\omega \xi) ; \phi_{3}=\cosh (\omega \xi) ; \phi_{4}=\sinh (\omega \xi) \\
& \phi_{5}=\cos \left(\omega\left(\xi-r_{1}\right)\right) ; \phi_{6}=\sin \left(\omega\left(\xi-r_{1}\right)\right) ; \phi_{7}=\cosh \left(\omega\left(\xi-r_{1}\right)\right) ; \phi_{8}=\sinh \left(\omega\left(\xi-r_{1}\right)\right) \\
& \phi_{9}=\cos (\omega(\xi-1)) ; \phi_{10}=\sin (\omega(\xi-1)) ; \phi_{11}=\cosh (\omega(\xi-1)) ; \phi_{12}=\sin h(\omega(\xi-1))
\end{aligned}
$$

Using the following orthogonality conditions:

$$
\begin{aligned}
& \int_{0}^{r_{2}} \cos \left(\bar{k}_{n} \xi\right) \cos \left(\bar{k}_{m} \xi\right) d \xi=0 \text { if } m \neq n \\
& \int_{0}^{r_{2}} \cos \left(\bar{k}_{n} \xi\right) \cos \left(\bar{k}_{m} \xi\right) d \xi=\frac{r_{2}}{2}+\frac{\sin \left(2 \bar{k}_{n} r_{2}\right)}{4 \bar{k}_{n}}=I_{n} \text { if } m=n
\end{aligned}
$$

and defining:

$$
I_{n j}=\int_{0}^{r_{2}} \cos \left(\bar{k}_{n} \xi\right) \phi_{j}(\xi) d \xi
$$

the following coefficients are obtained:

$$
\begin{aligned}
A_{n}^{(1)} & =E_{n} \sum_{j=1}^{4} D_{j} \operatorname{Im}_{j}(\xi) \\
A_{n}^{(2)} & =E_{n} \sum_{j=5}^{8} D_{j} \operatorname{Im}_{j}(\xi) \\
B_{n}^{(1)} & =\bar{E}_{n} \sum_{j=1}^{4} D_{j} \operatorname{Im}_{j}(\xi) \\
B_{n}^{(2)} & =\bar{E}_{n} \sum_{j=5}^{8} D_{j} \operatorname{Im}(\xi) \\
E_{n} & =\frac{1}{I_{n}\left[\frac{1}{\bar{k}_{n}^{4}-\omega^{4}}-\frac{\bar{\lambda}_{m}}{\gamma \omega^{4}}\right]} \\
\bar{E}_{n} & =\frac{1}{I_{n}\left[1-\frac{\bar{\lambda}_{m}\left(\bar{k}_{n} \omega^{4}\right)}{\gamma \omega^{4}}\right]}
\end{aligned}
$$

The substitution of these expressions into the boundary conditions Eqs (18d-18o) gives the following linear system with the associated eigenvalue problem:

$$
\begin{aligned}
R D & =0 \\
\operatorname{det} R & =0
\end{aligned}
$$

where $D$ is a vector of twelve elements and $R$ is a square matrix of which the non-zero parameters are:

$$
\begin{aligned}
R_{1 j} & =\phi_{j}(0)+\sum_{n=n_{1}}^{\infty} \bar{E}_{n} I_{n j} \\
j & =1,2,3,4
\end{aligned}
$$




$$
\begin{aligned}
& R_{2 j}=\phi_{j}^{\prime}(0) \\
& j=1,2,3,4 \\
& R_{3 j}=\phi_{j}^{\prime \prime}(1) \\
& j=9,10,11,12 \\
& R_{4 j}=\phi_{j}^{\prime \prime \prime}(1) \\
& j=9,10,11,12 \\
& R_{5 j}=\phi_{j}\left(r_{1}\right)+\sum_{n=n_{1}}^{\infty} \bar{E}_{n} I_{n j} f_{y_{n}}\left(r_{1}\right) \\
& j=1,2,3,4 \\
& R_{5 j}=-\phi_{j}\left(r_{1}\right)-\sum_{n=n_{1}}^{\infty} \bar{E}_{n} I_{n j} f_{y_{n}}\left(r_{1}\right) \\
& j=5,6,7,8 \\
& R_{6 j}=\omega \phi_{j}^{\prime}\left(r_{1}\right)+\sum_{n=n_{1}}^{\infty} \bar{E}_{n} I_{n j} f_{y_{n}}^{\prime}\left(r_{1}\right) \\
& j=1,2,3,4 \\
& R_{6 j}=-\omega \phi_{j}^{\prime}\left(r_{1}\right)-\sum_{n=n_{1}}^{\infty} \bar{E}_{n} I_{n j} f_{y_{n}}^{\prime}\left(r_{1}\right)-\frac{E J}{L k_{T}}\left(\omega^{2} \phi_{j}^{\prime \prime}\left(r_{1}\right)+\sum_{n=n_{1}}^{\infty} \bar{E}_{n} I_{n j} f_{y_{n}}^{\prime \prime}\left(r_{1}\right)\right) \\
& j=5,6,7,8 \\
& R_{7 j}=\omega^{2} \phi_{j}^{\prime \prime}\left(r_{1}\right)+\sum_{n=n_{1}}^{\infty} \bar{E}_{n} I_{n j} f_{y_{n}}^{\prime \prime}\left(r_{1}\right) \\
& j=1,2,3,4 \\
& R_{7 j}=-\omega^{2} \phi_{j}^{\prime \prime}\left(r_{1}\right)-\sum_{n=n_{1}}^{\infty} \bar{E}_{n} I_{n j} f_{y_{n}}^{\prime \prime}\left(r_{1}\right) \\
& j=5,6,7,8 \\
& R_{8 j}=\omega^{3} \phi_{j}^{\prime \prime \prime}\left(r_{1}\right)+\sum_{n=n_{1}}^{\infty} \bar{E}_{n} I_{n j} f_{y_{n}}^{\prime \prime \prime}\left(r_{1}\right) \\
& j=1,2,3,4 \\
& R_{8 j}=-\omega^{3} \phi_{j}^{\prime \prime \prime}\left(r_{1}\right)-\sum_{n=n_{1}}^{\infty} \bar{E}_{n} I_{n j} f_{y_{n}}^{\prime \prime \prime}\left(r_{1}\right) \\
& j=5,6,7,8 \\
& R_{9 j}=\phi_{j}\left(r_{2}\right)+\sum_{n=n_{1}}^{\infty} \bar{E}_{n} I_{n j} f_{y_{n}}\left(r_{2}\right) \\
& j=5,6,7,8 \\
& R_{9 j}=-\phi_{j}\left(r_{2}\right) \\
& j=9,10,11,12
\end{aligned}
$$




$$
\begin{aligned}
R_{10 j} & =\omega \phi_{j}^{\prime}\left(r_{2}\right)+\sum_{n=n_{1}}^{\infty} \bar{E}_{n} I_{n j} f_{y_{n}}^{\prime}\left(r_{2}\right) \\
j & =5,6,7,8 \\
R_{10 j} & =-\omega \phi_{j}^{\prime}\left(r_{2}\right) \\
j & =9,10,11,12 \\
R_{11 j} & =\omega^{2} \phi_{j}^{\prime \prime}\left(r_{2}\right)+\sum_{n=n_{1}}^{\infty} \bar{E}_{n} I_{n j} f_{y_{n}}^{\prime \prime}\left(r_{2}\right) \\
j & =5,6,7,8 \\
R_{11 j} & =-\omega^{2} \phi_{j}^{\prime \prime}\left(r_{2}\right) \\
j & =9,10,11,12 \\
R_{12 j} & =\omega^{3} \phi_{j}^{\prime \prime \prime}\left(r_{2}\right)+\sum_{n=n_{1}}^{\infty} \bar{E}_{n} I_{n j} f_{y_{n}}^{\prime \prime}\left(r_{2}\right) \\
j & =5,6,7,8 \\
R_{12 j} & =-\omega^{3} \phi_{j}^{\prime \prime \prime}\left(r_{2}\right) \\
j & =9,10,11,12
\end{aligned}
$$

Imposing that the determinant of $R$ is null, the adimensional frequency parameters of the coupled system are obtained.

The coefficients of the characteristic polynomial associated to the determinant depend on the adimensional frequency parameter when the fluid is compressible or when the effect of free surface is considered. In this case an iterative procedure is used for the solution.

\section{Numerical results}

Using the formulation described in the previous sections, numerical results have been obtained considering the following values for the geometry and the material of the beam coupled with the fluid: $h=1 \mathrm{~m}, b=1 \mathrm{~m}$, $\gamma=10, r_{2}=0.8$, Young's modulus $E=2.94 \mathrm{e}^{10} \mathrm{~Pa}$, density of the beam $\rho_{S}=2400 \mathrm{~kg} / \mathrm{m}^{3}$, density of the fluid $\rho_{F}=1000 \mathrm{~kg} / \mathrm{m}^{3}$.

For each of these cases, the trend of the first and the second frequency parameters has been plotted as a function of the crack position $\left(r_{1}\right)$ for different ratios a/h relatively to the following cases:

- uncompressible fluid without sloshing (Figs 2 and 3);

- compressible fluid without sloshing (Figs 4 and 5);

- compressible fluid with sloshing (Figs 6 and 7).

Here the parameters have been obtained by annulling the determinant of matrix $R$ and, for the case relative to the free surface proceeding through an iterative calculation due to the dependence of $\left(k_{n}\right)$ on the frequency. When an undamaged uncompressible beam without sloshing is considered the adimensional frequency parameters obtained by [41] have been determined $\left(\omega_{1}=1.6273, \omega_{2}=3.6610\right)$.

As regards the cases with sloshing and compressible fluid, a comparison with the work of [41] has not been made as it is not possible to define an adimensional frequency parameter which is independent of the geometry of the beam.

While in the case of incompressible fluid without sloshing there is a decrease of the natural frequencies and consequently the fluid can be considered as an additional mass, the presence of compressibility and of the free 
surface gives a contribution in term of potential energy (due to the deformations of the fluid and the elevation of the centre of gravity of the water mass) and in terms of kinetic energy.

Figures 4 and 5, relative to the case of compressible fluid without sloshing, practically coincide with Figs 2 and 3 , as the compressibility of the fluid chosen for the calculation (water) is very low.

The effect of the sloshing appears to have greater influence when the frequencies are low, as has been observed in [41]. The effect of compressibility in the example is not obvious due to the low value of the compressibility of the fluid considered. However, coherently with the work of Xing et alii, the effect exhibited is more evident at higher frequencies.

In order to check the validity of the results ,the case relative to the beam in absence of fluid has been studied. In this case the adimensional frequencies coincide with those already known relative to cracked beams in vacuum (Figs 8 and 9). The first frequency parameter trend is increasing when the crack is further from the clamped end. For successive frequencies the trend is not monotone as has been demonstrated in past studies.

\section{Conclusion}

In this the flexural behaviour of a cracked dam modelled with a prismatic beam in contact with a compressible fluid with free surface has been analysed, the crack being represented by a massless rotational spring.

Following the approach in ref. [41], the coupled beam-water eigen-problem has been solved using the method of variable-separation. An exact solution has been obtained for several boundary conditions: incompressible fluid without sloshing, a compressible fluid without and with free surface.

In order to evaluate the effect of damage in the fluid-structure interaction problem, different crack sizes and positions have been considered. Finally the case of the cracked beam in vacuum has been studied to compare the results with those present in literature.

\section{References}

[1] M. Amabili, Comments on The Rayleigh Ritz method in coupled fluid interaching systems and its application, Journal of Sound and Vibration 195 (1996), 346-347.

[2] M. Amabili, Effect of finite fluid depth on the hydroelastic vibration of circular and annular plates, Journal of Sound and Vibration 193 (1996), 909-925.

[3] M. Amabili, G. Frosali and M.K. Kwak, Free vibrations of circular plates coupled with liquids: revising the Lamb problem, Journal of Fluids and Structures 10 (1996), 743-761.

[4] M. Amabili and M.K. Kwak, Free vibrations of annular plates coupled with fluid, Journal of Sound and Vibration 191 (1996), 825-846.

[5] M. Amabili, A. Pasqualini and G. Dalpiaz, Natural frequencies and modes of free-edge circular plates vibrating in vacuum or in contact with liquid, Journal of Sound and Vibration 188 (1995), 685-699.

[6] M. Amabili, Ritz method and substructuring in the study of vibration with strong fluid structure interaction, Journal of Fluids and Structures 11 (1997), 507-523.

[7] H.F. Bauer, Hydroelastic vibrations in rectangular container, International Journal of Solids and Structures 17 (1981), 639-652.

[8] Y.K. Cheung, Z. Cao and S.Y. Wu, Dynamic analysis of prismatic structures surrounded by an infinite fluid medium, Earthquake Engineering and Structural Dynamics 13 (1985), 351-360.

[9] M. Chiba, Nonlinear hydroelastic vibration of a cylindrical tank with an elastic bottom, containing liquid. Part II: linear axisymmetric vibration analysis, Journal of Fluids and Structures 7 (1993), 57-73.

[10] T.G. Chondros and A.D. Dlmarogonas, Identification of cracks in welded joints of complex structures, Journal of Sound and Vibration $\mathbf{6 9}$ (1980), 531-538.

[11] S. Christides and A.D.S. Barr, One-dimensional theory of cracked Euler-Bernoulli beams, International Journal of Mechanical Science 26 (1984), 639-648.

[12] D. Dlmarogonas, Vibration of cracked structures: a state of the art review, Engineering Fracture Mechanics 55 (1996), 831-857.

[13] S.W. Doebling, C.R. Farrar, M.B. Prime and D.W. Shevitz, Report no. LA-13070-MS, Los Alamos National Laboratory, Damage identification and health monitoring of structural and mechanical systems from changes in their vibration characteristics: a literature review, 1996.

[14] Y. Fu and W.G. Price, Interactions between a partially or totally immersed vibration cantilever plate and the surrounding fluid, Journal of Sound and Vibration 118 (1987), 495-513.

[15] G. Gounaris and A.D. Dimarogonas, A finite-element of a cracked prismatic beam for a structural analysis, Computer and Structures 28 (1988), 309-313. 
[16] P. Gudmunson, The dynamic behaviour of slender structures with cross-sectional cracks, Journal of Mechanics and Physics of Solids 31 (1993), 329-345.

[17] S. Hylarides and W.S. Vorus, The added mass matrix in ship vibration, using a source distribution related to the finite element grid of the ship structure, International Shipbuilding Progress 29 (1982), 34-43.

[18] J. Hu and R.Y. Liang, An integrated approach to detection of cracks using vibration characteristics, Journal of the Franklin Institute 330 (1993), 841-853.

[19] K.H. Jeong, Dynamics of a concentrically or eccentrically submerged circular cylindrical shell in a fluid filled container, Journal of Sound and Vibration 224(4) (1999) 709-732.

[20] K.H. Jeong and K.A. Kim, Free vibration of a circular cylindrical shell filled with bounded compressible fluids, Journal of Sound and Vibration 217(2) (1998), 197-221.

[21] K.H. Jeong, Natural frequencies and mode shapes of two coaxial cylindrical shells coupled with compressible fluids, Journal of Sound and Vibration 215(1) (1998), 105-124.

[22] A. Joshi and B.S. Madhusudhan, A unified approach to free vibration of locally damaged beams having various homogeneous boundary conditions, Journal of Sound and Vibration 147 (1991), 475-488.

[23] F. Kito, Principles of Hydroelasticity, Tokyo: Keio University, 1970.

[24] M.K. Kwak, Hydroelastic Vibration of a rectangular plates, Journal of Applied Mechanics 63 (1996), 110-115.

[25] H.B. Lamb, On the vibrations of an elastic plate in contact with water, Proceeding of Royal Society of London A 98 (1921), 205-216.

[26] H.B. Lamb, Hydrodynamics, Dover Inc, 1945.

[27] R.Y. Liang, J. Hu and F. Choy, Theoretical study of crack-induced eigen-frequency changes on beam structures, Journal of Engineering Mechanics 118 (1992), 384-396.

[28] R.Y. Liang, J. Hu and F. Choy, Quantitative NDE techniques for assessing damages in beam structures, Journal of Engineering Mechanics 118 (1992), 1468-1487.

[29] R.Y. Liang, F. Choy and J. Hu, Detection of cracks in beam structures using measurements of natural frequencies, Journal of the Franklin Institute 328 (1991), 505-518

[30] M.S. Marcus, A finite element method applied to the vibration of submerged plates, Journal of Ship Research 22 (1978), 94-99.

[31] C. Mares and C. Surace, An application of genetic algorithm to identify damage in elastic structures, Journal of Sound and Vibration 195 (1996), 195-215

[32] Y.W.M. Ostachowicz and M. Krawczuk, Analysis of the effect of cracks on the natural frequencies of a cantilever beam, Journal of Sound and Vibration 150 (1991), 191-201.

[33] G.L. Qian, S.N. Gu and J.S. Jiang, The dynamic behaviour and crack detection of a beam with a crack, Journal of Sound and Vibration 138 (1990), 233-243.

[34] H. Rapoport, Dynamics of Elastic Containers, New York: Springler and Verlag, 1968.

[35] P.F. Rizos, N. Aspragatos and A.D. Dimarogonas, Identification of crack location and magnitude in a cantilever beam from the vibration modes, Journal of Sound and Vibration 138 (1990), 381-388.

[36] R. Ruotolo and C. Surace, Damage assessment of multiple cracked beams: numerical results and experimental validation, Journal of Sound and Vibration 206 (1997), 567-588.

[37] R. Ruotolo, C. Surace and C. Mares, Proceedings of 14th International Modal Analysis Conference, Theoretical and experimental study of the dynamic behaviour of a double-cracked beam, 1996, pp. 1560-1564.

[38] R. Ruotolo, C. Surace and C. Mares, Proceedings 15th International Modal Analysis Conference, Damage identification using simulated annealing, 1997, pp. 954-960.

[39] W. Soedel, On the natural frequencies and modes of beams loaded by sloshing liquids, Journal of Sound and Vibration 85 (1982), 345-353.

[40] W. Soedel and M. Soedel, On the natural frequencies and modes of beams loaded by sloshing liquids, Journal of Sound and Vibration $\mathbf{8 5}$ (1994), 345-353.

[41] J.T. Xing, W.G. Price, M.J. Pomfret and L.H. Yam, Natural vibration of a beam water interaction system, Journal of sound and vibration 199(3) (1997), 491-512.

[42] M.M. Yuen, A numerical study of the eigen-parameters of a damaged cantilever, Journal of Sound and Vibration 103 (1985), 301-310. 

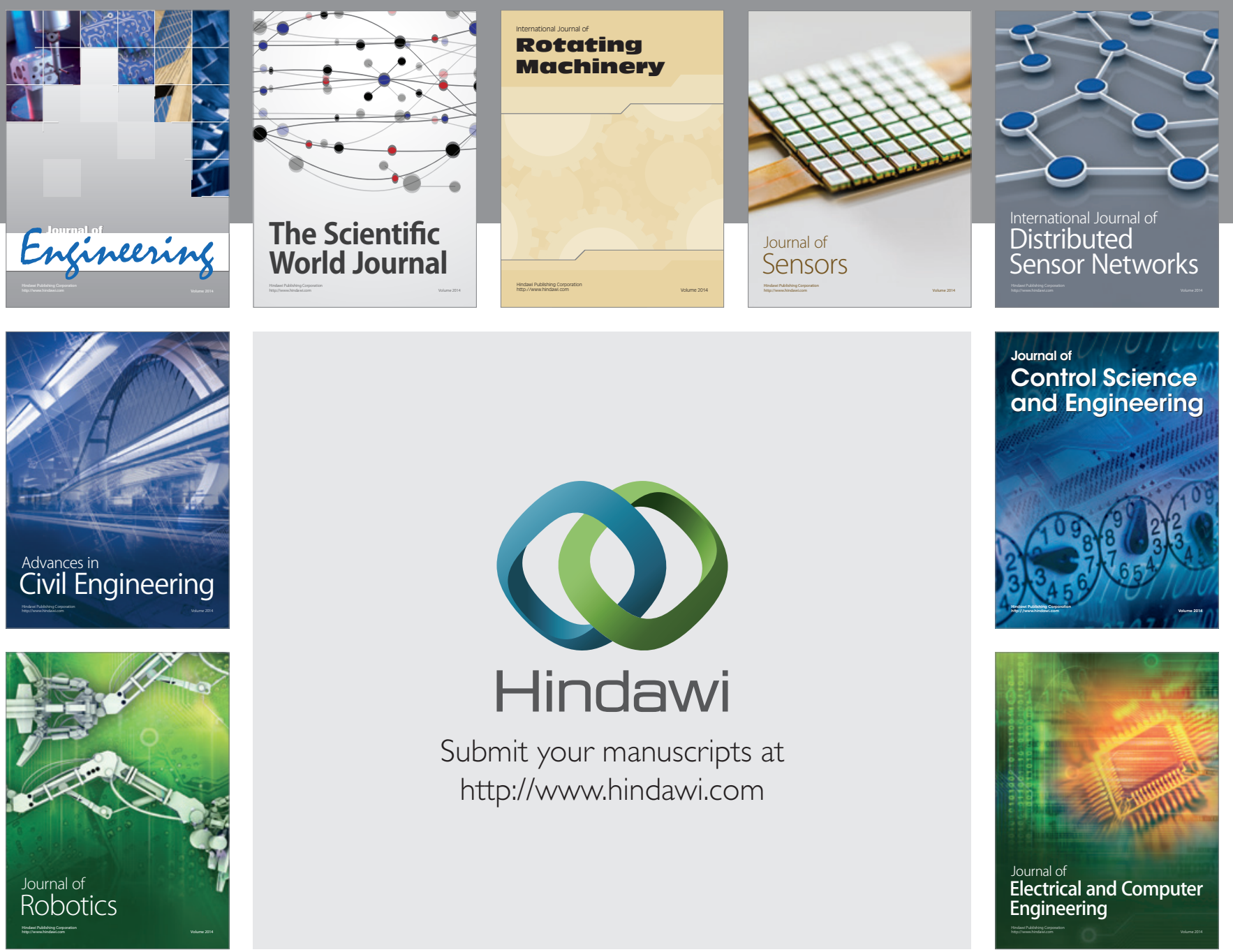

Submit your manuscripts at

http://www.hindawi.com
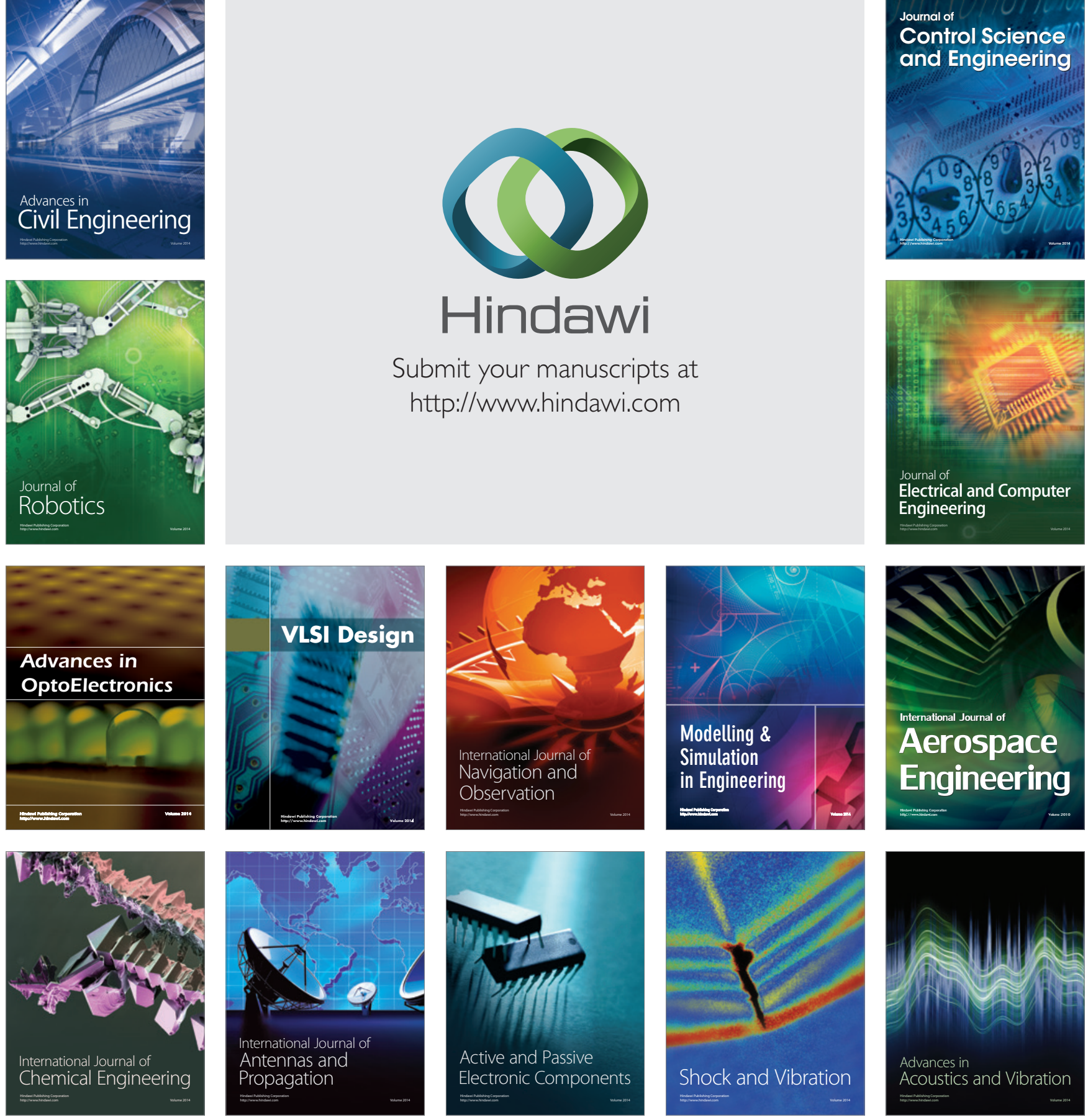